\title{
Hajós-Property for Direct Product of Groups
}

\author{
Khalid Amin \\ Department of Mathematics, University of Bahrain, Sakhir, Kingdom of Bahrain \\ Email:kameen@uob.edu.bh
}

Received 23 July 2015; accepted 16 November 2015; published 19 November 2015

Copyright () 2015 by author and Scientific Research Publishing Inc.

This work is licensed under the Creative Commons Attribution International License (CC BY). http://creativecommons.org/licenses/by/4.0/

(c) (i) Open Access

\begin{abstract}
We study decomposition of finite Abelian groups into subsets and show by examples a negative answer to the question of whether Hajós-property is inherited by direct product of groups which have Hajós-property.
\end{abstract}

\section{Keywords}

\section{Abelian Groups, Hajós-Property, Factorization of Abelian Groups}

\section{Introduction}

The general setting is as follows: Suppose we decompose a group $G$ into direct product of subsets $A_{1}, A_{2}, \cdots, A_{n}$ of $G$ in such a way that each element $g$ in $G$ has a unique representation of the form $g=a_{1} a_{2} \cdots a_{n}$, where $a_{i} \in A_{i}$. The question then asked is what we can say about the subsets $A_{1}, A_{2}, \cdots, A_{n}$.

The answer is rather difficult even if we do not impose many restrictions either on $G$ or on the subsets. The most important special case has some connection with a group-theoretial formulation by G. Hajós [1] of a conjecture by $\mathrm{H}$. Minkowski [2]; this is when $G$ is a finite Abelian group and each of the subsets is of the form

$$
A_{i}=\left\{e, g, g^{2}, \cdots, g^{k}\right\},
$$

where $k<|g|$ is an integer; here $e$ denotes the identity element of $g$ and $|g|$ denotes order of the element $g$ of $G$. Then a result due to Hajos states that one of the subsets $A_{\text {i }}$ must be a subgroup of $G$. L. Rédei [3] generalizes this result to the case when the condition on the subsets $A_{i}$ is that they contain a prime number of elements.

Another interesting question has also been asked by Hajos. It is concerned with the case in which $G$ is an Abelian group and $n=2$; the question then asked is as follows: Suppose $G$ has a decomposition as $G=A_{1} A_{2}$. Does it follow that one of the subsets $A_{1}$ or $A_{2}$ is a direct product of another subset and a proper subgroup of $G$ ? 
The concept of Hajós factorization begin group-theoretical but now finds applications in diverse fields such as number theory, [4] coding theory [5] and even in music [6].

\section{Preliminaries}

Throughout this paper, $G$ will denote a finite Abelian group, $e$ the identity of $G$, and if $g \in G$, then $|g|$ will denote its order. We will also use $|A|$ to denote the number of elements of a subset $A$ of $G$. A subset $A$ of $G$ of the form $A=\left\{e, g, g^{2}, \cdots, g^{k}\right\}$ is called a cyclic subset of $G$; here $k$ is an integer with $k<|g|$. If

$$
G=A_{1} A_{2} \cdots A_{n}
$$

we say that we have a factorization of $G$. If in addition, each of the subsets $A_{i}$ contains $e$, we say that we have a normalized factorization of $G$. A subset $A$ of $G$ is called periodic if there exists $g \in G-\{e\}$, such that $g A=A$. Such an element $g \in G$ if it exists is called a period for $A$. A group $G$ is said to be of type $\left(p_{1}^{\alpha_{1}}, p_{2}^{\alpha_{2}}, \cdots, p_{r}^{\alpha_{r}}\right)$, if it is a direct product of cyclic groups of orders $p_{1}^{\alpha_{1}}, p_{2}^{\alpha_{2}}, \cdots, p_{r}^{\alpha_{r}}$, (where of course $p_{i}^{\prime} s$ are primes and $\alpha_{i}^{\prime}$ s are non-negative integers).

\section{Remarks}

1) If $G=A B$ is a factorization of $G$, then for any $x, y \in G, x A y B=G$ is also a factorization of $G$. Similarly, with $G=A_{1} A_{2} \cdots A_{n}$. Thus, we may assume that all factorization we consider are normalized.

2) In the literature, a group $G$ is said to be "good" if from each factorization $G=A B$, it follows that one the subsets $A$ or $B$ is periodic.

We extend the above definition as follows.

\section{Definition}

A group $G$ has the Hajos-n-property or $n$-good if from any factorization

$$
G=A_{1} A_{2} \cdots A_{n}
$$

it follows that one of the subsets $A_{1}, A_{2}, \cdots, A_{n}$ is periodic. Otherwise it is $n$-bad. We will also say $G$ is totallygood if it is $n$-good for all possible values of $n$.

The following results are known and will be used in this paper.

Lemma 1 [7]

If $G$ is of type $\left(2^{2}, 2^{2}\right)$, then $G$ is 2-good.

Lemma 2 [8]

A cyclic group $G$ of order $p^{\alpha}$, where $p>3$ is prime is totally-good.

Lemma 3 [8]

If $G$ is of type $\left(p^{\alpha}, p^{\beta}\right)$, where $1 \leq \alpha \leq \beta, \beta \geq 2$ and $p>3$ is prime, then $G$ is $n$-bad for all $n$, $2 \leq n \leq \alpha+\beta-1$.

Lemma 4 [9]

If $H$ is a proper subgroup of $G$, then there exists a non-periodic set $N$ such that $G=H N$ is a factorization of $G$, except when $H$ is a subgroup of index 2 in an elementary abelian 2-group.

\section{Lemma 5 [7]}

If $A$ and $B$ are non-periodic subsets o a group $G$ and $A$ is contained in a subgroup $H$ of $G$ such that $G=H B$ is a factorization of $G$, then $A B$ is also non-periodic.

\section{Results}

\section{Theorem 6}

If $G$ is of type $\left(2^{2}, 2^{2}\right)$, then $G$ is totally-good.

Proof.

Let $G=A_{1} A_{2} \cdots A_{n}$ be a factorization of $G$.

Now, the possible values for $n$ are 1, 2, 3 and 4 .

The case $n=1$ is trivial.

The case $n=2$ follows from Lemma 1. 
The case $n=4$ follows from Rédei's theorem.

So, we only need details the case $n=3$. So now, $G=A_{1} A_{2} A_{3}$.

We may assume $\left|A_{1}\right|=\left|A_{2}\right|=2$. Now, $G=A_{1}\left(A_{2} A_{3}\right)$ is also a factorization of $G$. Hence by Lemma 1, either $A_{1}$ is or $A_{2} A_{3}$ is periodic. If $A_{1}$ is periodic, we are done. So assume $A_{2} A_{3}$ is periodic, say with period $g \neq e$. We may assume $|g|=2$.

Let $A_{2}=\{e, x\}$ and $A_{3}=\{e, y\}$. Then $A_{2} A_{3}=\{e, x, y, x y\}$. If $g=x$, then $A_{2}$ is a subgroup and hence periodic, while if $g=y$, then $A_{3}$ is a subgroup and hence periodic. Suppose $g=x y$, then we must have either 1) $x^{2} y=x$ and $x y^{2}=y$ both of which give $x y=e$, which is impossible; or 2) $x^{2} y=y$ and $x y^{2}=x$ both of which imply that both $A_{1}$ and $A_{2}$ are subgroups of $G$. This ends the proof.

\section{Theorem 7}

If $G$ is of type $\left(2^{2}, 2^{2}, 2\right)$, then $G$ is 3-bad.

Proof.

Let $G=\langle x\rangle \times\langle y\rangle \times\langle z\rangle$, where $|x|=|y|=4$ and $|z|=2$.

Let $A_{1}=\{e, x\}, A_{2}=\{e, y\}$ and $A_{3}=\left\{e, x^{2}, x y^{2}, x^{3} y^{2}, z, y^{2} z, x^{2} y z, x^{2} y^{3} z\right\}$.

Then $G=A_{1} A_{2} A_{3}$ is a factorization of $G$ and none of the subsets $A_{1}, A_{2}$ or $A_{3}$ is periodic. This ends the proof.

\section{Theorem 8}

Let $H$ be a proper subgroup of a group $G$. If $H$ is $n$-bad, then $G$ is both $n$ and $(n+1)$-bad.

Proof.

Since $H$ is $n$-bad, there is a factorization $H=A_{1} A_{2} \cdots A_{n}$ of $H$, where none of the subsets $A_{1}, A_{2}, \cdots, A_{n}$ is periodic. Now, by Lemma 5, there is a factorization $G=H A_{n+1}$ of $G$, with $A_{n+1}$ nonperiodic. Hence,

$$
G=A_{1} A_{2} \cdots A_{n} A_{n+1}
$$

is a factorization $G$ with none none of the subsets $A_{1}, A_{2}, \cdots, A_{n}, A_{n+1}$ periodic. Thus, $G$ is $(n+1)$-bad.

Also, $G=A_{1} A_{2} \cdots A_{n-1}\left(A_{n} A_{n+1}\right)$ is a factorization $G$ with none of the subsets periodic. Here, the nonperiodicity of the factor $\left(A_{n} A_{n+1}\right)$ follows from Lemma 5 . This ends the proof.

\section{Theorem 9}

If $G$ is of type $\left(2^{\alpha_{1}}, 2^{\alpha_{2}}, \cdots, 2^{\alpha_{r}}\right)$, where $r \geq 3, \alpha_{1}, \alpha_{2} \geq 2$, then $G$ is both 3 and 4-bad.

Proof.

$G$ has a subgroup $H$ of type $\left(2^{2}, 2^{2}, 2\right)$ which is 3-bad by Theorem 7 .

So, the result follows from Theorem 8 . This ends the proof.

Finally, we show by example what we aimed to show.

\section{Example 1}

Let $G_{1}$ be of type $\left(2^{2}, 2^{2}\right)$. Then by Lemma 1, $G_{1}$ is 2-good. Now, consider the group $G=G_{1} \times G_{1}$ and note that $G$ is of type $\left(2^{2}, 2^{2}, 2^{2}, 2^{2}\right)$ Observe that $G$ has a subgroup $H$ of type $\left(2^{2}, 2^{2}, 2\right)$ which is 3-bad by Theorem 9. Now, by Lemma 4, $G$ has a factorization $G=H N$, where $N$ is nonperiodic. Hence, $G$ has a factorization $G=A_{1} A_{2} A_{3} N$, where none of the factor is periodic. Thus $G$ is 4-bad. This ends the proof.

\section{Example 2}

Let $G_{1}$ be of type $\left(p^{\alpha}\right)$ and $G_{2}$ be of type $\left(p^{\beta}\right)$, where $\alpha$ and $\beta$ are positive integers and $p>3$ is prime. Then by Lemma 2, $G_{1}$ is $m$-good for all $m, 1 \leq m \leq \alpha$, and $G_{2}$ is $n$-good for all $m, 1 \leq n \leq \beta$. Consider the group $G=G_{1} \times G_{2}$. Then by Lemma 3, $G_{1}$ is $(m+n)$-bad for all $m+n, 2 \leq m+n \leq \alpha+\beta$. This ends the proof.

\section{References}

[1] Minkowski, H. (1896) Geometrie der Zahlen. Teubner, Leipzig.

[2] Hajos, G. (1949) Sur la factorsation des groups aeliens. Casopis, 74, 157-162.

[3] Rédei, L. (1965) Die neue Theoreie der endlichen abelschen Gruppen und Verall-geomeinerung des Hauptsatze von Hajós. Acta Mathematica Academiae Scientiarum Hungaricae, 16, 329-373. http://dx.doi.org/10.1007/BF01904843

[4] De Bruijn, N.G. (1950) On Bases for the Set of Integers. Publicationes, Mathematicae, 232-242. 
[5] Amin, K. (2014) Constructing Single-Error-Correcting Codes Using Factorization of Finite Abelian Groups. International Journal of Algebra, 8, 311-315.

[6] Vuza, D.T. (1990-91) Supplementary Sets and Regular Complementary Unending Canons. Perspectives of New Music, 23.

[7] Sands, A.D. (1962) Factorization of Abelian Groups. The Quarterly Journal of Mathematics, 13, 45-54. http://dx.doi.org/10.1093/qmath/13.1.45

[8] Amin, K. (1997) The Hajos-n-Property for finite $p$-Groups. PUMA, 1-12.

[9] Sands, A.D. (1959) Factorization of Abelian Groups. The Quarterly Journal of Mathematics, 10, 81-91. http://dx.doi.org/10.1093/qmath/10.1.81 